\title{
The correlation between posterior tibial slope and dynamic anterior tibial translation and dynamic range of tibial rotation
}

\author{
M.J.M Zee ${ }^{1 *}$, M.N.J Keizer² ${ }^{2}$ L Dijkerman ${ }^{1}$, J.J.A.M van Raaij ${ }^{3}$, J.M. Hijmans ${ }^{4}$ and R.L. Diercks ${ }^{1}$
}

\begin{abstract}
Purpose: The amount of passive anterior tibial translation (ATT) is known to be correlated to the amount of posterior tibial slope (PTS) in both anterior cruciate ligament-deficient and reconstructed knees. Slope-altering osteotomies are advised when graft failure after anterior cruciate ligament $(\mathrm{ACL})$ reconstruction occurs in the presence of high PTS. This recommendation is based on studies neglecting the influence of muscle activation. On the other hand, if dynamic range of tibial rotation (rTR) is related to the amount of PTS, a "simple" anterior closing-wedge osteotomy might not be sufficient to control for tibial rotation. The purpose of this study was to evaluate the correlation between the amount of PTS and dynamic ATT and tibial rotation during high demanding activities, both before and after ACL reconstruction. We hypothesized that both ATT and rTR are strongly correlated to the amount of PTS.

Methods: Ten subjects were studied both within three months after ACL injury and one year after ACL reconstruction. Dynamic ATT and dynamic rTR were measured using a motion-capture system during level walking, during a single-leg hop for distance and during a side jump. Both medial and lateral PTS were measured on MRI. A difference between medial and lateral PTS was calculated and referred to as $\triangle$ PTS. Spearman's correlation coefficients were calculated for the correlation between medial PTS, lateral PTS and $\triangle$ PTS and ATT and between medial PTS, lateral PTS and $\triangle \mathrm{PTS}$ and $r T R$.
\end{abstract}

Results: Little (if any) to weak correlations were found between medial, lateral and $\triangle$ PTS and dynamic ATT both before and after $A C L$ reconstruction. On the other hand, a moderate-to-strong correlation was found between medial PTS, lateral PTS and $\triangle$ PTS and dynamic rTR one year after ACL reconstruction.

Conclusion: During high-demand tasks, dynamic ATT is not correlated to PTS. A compensation mechanism may be responsible for the difference between passive and dynamic ATT in terms of the correlation to PTS. A moderate-tostrong correlation between amount of PTS and rTR indicates that such a compensation mechanism may fall short in correcting for rTR. These findings warrant prudence in the use of a pure anterior closing wedge osteotomy in $\mathrm{ACL}$ reconstruction.

Trial registration: Netherlands Trial Register, Trial 7686. Registered 16 April 2016-Retrospectively registered.

Level of evidence: Level 2, prospective cohort study

Keywords: Anterior Cruciate Ligament (ACL), ACL reconstruction, Tibial rotation, Anterior tibial translation, Posterior tibial slope

\footnotetext{
*Correspondence: m.j.m.zee@umcg.nl

${ }^{1}$ Department of Orthopaedic Surgery, University Medical Center Groningen, PO Box 30.001, 9700 RM Groningen, The Netherlands Full list of author information is available at the end of the article
}

\section{Background}

Risk factors for ACL injury are multifactorial and, next to gender-related, genetic, and hormonal factors, include anatomical and biomechanical factors [14, 
25]. One anatomical factor that has been of interest in recent studies is the amount of posterior tibial slope (PTS). From cadaveric experiments it is known that increased PTS leads to more forward-directed forces on the tibia and increases strain on the ACL [3]. Dejour and Bonnin showed that every increase of $10^{\circ}$ in PTS leads to a $6 \mathrm{~mm}$ increment of passive anterior tibial translation (ATT) in ACL deficiency [10]. More recent studies confirm the correlation between PTS and passive ATT in both ACL-deficient and ACL-intact knees $[8,9,13]$.

Increased PTS is related to increased risk of primary ACL injury and increased risk of graft failure after ACL reconstruction $[6,30,32]$. For this reason it has been suggested that, in revision cases, altering the amount of PTS by an anterior closing-wedge osteotomy could reduce strain on the ACL graft and prevent another reinjury [17]. It should be noted that past studies have evaluated passive ATT either using instrumented Lachman or in a cadaveric setting, both of which eliminate muscle tone. The influence of PTS on dynamic ATT is less extensively studied.

As clearly as the relation between PTS and passive ATT is demonstrated, less is known about the relation between PTS and tibial rotation. The ACL is known to restrict $\mathrm{ATT}$, but also plays a role in limiting tibial rotation [12]. Due to the anatomical features of the tibial plateau, axial load transfers into an anteriorly directed force on the tibia [10]. This force increases with PTS [10]. As the medial and lateral tibial plateaus differ in congruency with the femur, as well as in mobility, we argue that the translation in the lateral compartment is more susceptible to changes in PTS. Due to this difference between the medial and the lateral compartment, axial load would not only be transferred into ATT, but also into rotation of the tibia relative to the femur. We hypothesized that this difference (referred to as $\triangle \mathrm{PTS}$ ) might be of more importance than the actual amount of slope itself, with respect to rotation.

If the range of tibial rotation (rTR) is related to the amount of PTS, a "simple" anterior closing-wedge osteotomy might not be sufficient to control for tibial rotation.

The aim of this study was to answer the following research questions:

- Is PTS correlated to dynamic ATT before and after ACL reconstruction?

- Is $\triangle \mathrm{PTS}$ correlated to $\mathrm{rTR}$ before and after ACL reconstruction?

We hypothesized that both ATT and rTR are strongly correlated to the amount of $(\Delta)$ PTS.

\section{Methods}

To answer the research questions, subjects with acute ACL injury were kinematically assessed using in vivo kinematic motion analysis. Dynamic ATT and rTR were measured during level walking, a single-leg hop for distance (SLHD) and a side jump. This study was set up as a multicentre prospective cohort study. Both University Medical Center Groningen (UMCG) and Martini Hospital (Groningen, the Netherlands) included subjects in the study. The study protocol was reviewed and approved by the institutional review board of the UMCG (ID 2015/524). The study was registered in the Dutch Trial Register (NTR: www.trialgregister.nl, registration ID NL7686). From June 2016 to June 2018 all patients diagnosed with ACL injury in one of the two participating hospitals were screened for eligibility to participate in the study. Inclusion criteria were: (1) age 18-35 years, (2) unilateral ACL injury confirmed by physical examination, (3) less than three months post- injury at time of diagnosis, (4) at least six weeks of conservative therapy, (5) intact contralateral knee on physical examination. Exclusion criteria were: (1) any history of fractures, osteotomy, or previous ligament reconstructive surgery in the lower extremities or spine, (2) neurological conditions leading to musculoskeletal disorders, (3) any other musculoskeletal pathology of the lower limbs (i.e. concomitant ligament or meniscal injuries), (4) inability to complete Dutch-language questionnaires.

\section{Surgical procedure}

All subjects underwent anatomic, single-bundle ACL reconstruction using a semitendinosus/gracilis graft. Both tendons were doubled to create a 4-strand graft. For femoral fixation a suspension type fixation was used (Endobutton, Smith\&Nephew, London, UK). After pretensioning $(60 \mathrm{~N})$, tibial fixation was performed by using a PEEK screw and plug (Biosure PK, Smith\&Nephew, London, UK).

\section{Data collection}

The motion data collection was performed at the motion lab of the UMCG's department of Rehabilitation Medicine. The motion lab consists of a $9 \mathrm{~m}$ walkway with two $40 \times 60 \mathrm{~cm}$ force plates (AMTI; Watertown, MA) embedded in the floor. An 8-camera optoelectronic motion capture system (VICON MX, Vicon Motion Systems Ltd., Oxford, UK) sampling at $100 \mathrm{~Hz}$ was used. The position of $2214 \mathrm{~mm}$ spherical markers, distributed on the lower extremities according to Hayes and Davis, was recorded [7]. After static and dynamic calibration, joint centres were calculated using VICON Nexus software v2.8 
(VICON MX, Vicon Motion Systems Ltd., Oxford, UK). For the complete procedure and its sensitivity see Keizer and Otten (2020) [19].

All subjects performed three tasks: (1) level walking at a self-selected pace; (2) a single-leg hop for distance (SLHD, maximum forward jump, jumping from and landing on the same leg); and (3) side jump (maximum sideways jump, jumping from and landing on the same leg). All jump trials were performed with hands in free motion and with sport shoes on. To familiarize subjects with the procedure and to make sure the entire foot would land on the force plate, subjects were asked to perform a dry run of the SLHD consisting of three practice trials. The median of the three practice hops was used to determine the starting distance from the force plates. For the side jump, leg length (greater trochanter tip to lateral malleolus tip) was used as starting distance from the centre of the force plates. Trials were included in the analysis when tasks were performed correctly (i.e. stable landing), the entire foot landed on the force plate, and all markers were left in place. Three correct trials were recorded for each leg. ACL-deficient subjects were tested within three months after injury. Approximately 13 months after the first trial, 12 months after ACL reconstruction, the testing procedure was repeated.

\section{Data processing}

The positions of the markers provided data to determine pelvis, femoral, tibial and foot segments. Using VICON Nexus software v2.8 and additional custom MATLAB version 9.7 scripts (The MathWorks Inc., Natick, MA, USA), three dimensional angular displacements and translations in the knee joint were calculated. Data processing and analysis started at initial contact and continued for $200 \mathrm{~ms}$. Initial contact was defined as the moment at which the vertical ground-reaction force (GRF) was $>5 \%$ of the body weight. All data were smoothed using the cross-validated quintic spline. Raw 3D marker position data were filtered by using a low pas frequency convolution filter of $10 \mathrm{~Hz}$ with zero lag. A maximum gap (temporary absence of marker identification) of 10 frames was accepted to fill in using the software. If a trial contained gaps exceeding $2.5 \mathrm{~ms}$, smoothing of the data could not be performed and trials were discarded. Kinematic variables were quantified and included maximum knee flexion, maximum knee extension, maximum knee valgus, maximum knee varus, maximum anterior tibial translation, range of tibial rotation, and knee flexion moment. Knee flexion moment was calculated from the GRF vector and its lever arm to the center of the knee flexion axis of the stance leg. For quantification of ATT, rTR and knee angles, two coordinate systems were reconstructed in the tested leg using the customized MATLAB script based on the method of Boeth et al. [4] One system was reconstructed in the femoral segment (parent system) and one in the tibial segment (child system). The motion of each coordinate system is consistent with the movement of the respective segment. The ATT was quantified in millimeters using the relative movement of the center of rotation of the tibial coordinate system relative to the center of rotation of the femoral coordinate system in the local tibial coordinate system. The range of tibial rotation was quantified by the angle between the two axes of rotation as outlined by Keizer and Otten [19]. Flexion/extension and varus/valgus angles were obtained using scalar products as in the equations explained by Robertson et al. [26]

\section{Measurement of PTS}

As part of usual care, all subjects underwent magnetic resonance imaging (MRI) of the injured knee to exclude concomitant injury. The images were used to calculate medial and lateral PTS using the circle method as described by Hudek et al. [15] A customized MATLAB script (The MathWorks, Inc., Natick, MA, USA) was used to measure both medial and lateral PTS on MRI. Two independent observers measured both medial and lateral PTS on all MRIs twice, with a minimum two-week interval. To determine intra- and interobserver reliability of the PTS measurements, intraclass correlation coefficients (ICC, 2-way random, absolute agreement) were calculated. Values lower than 0.5 were considered indicative of poor reliability, values between 0.5 and 0.75 indicated moderate reliability, between 0.75 and 0.9 good reliability, and greater than 0.90 excellent reliability [22].

\section{Statistical analysis}

Statistical analysis was performed using SSPS (v 23; IBM Corp, Armonk, NY, USA). A general linear model was used to test for differences between the three trials. Means were calculated for each subject over the three trials to obtain one value for ATT and rTR for each movement. A mean value of medial and lateral PTS from both observers and both measurements was used for analysis.

To assess the correlation between PTS and ATT and between PTS and rTR, Spearman's correlation coefficients were calculated. This was performed for medial PTS, lateral PTS and $\triangle$ PTS. Correlation coefficients were interpreted according to criteria set by Domholdt et al.: $0.00-0.25$ represents little if any correlation; 0.26-0.49 weak correlations; $0.50-0.69$ moderate; $0.70-0.89$ strong; and $0.90-1.00$ very strong correlations [11]. To reduce the effect of multiple testing, statistical tests deemed significant if $P<0.02$. 


\section{Results}

A total of 394 subjects were diagnosed with ACL injury and screened for eligibility. Fifty-seven subjects matched the inclusion criteria and were invited to participate in the study. Eleven subjects provided informed consent and were included in the study. The data of one subject was not used for analysis due to the subject's inability to perform the jumping tasks at the initial session. Six males and four females $(\mathrm{N}=10)$ completed the baseline testing procedures. At follow-up, 12 months after surgery seven subjects remained $(\mathrm{N}=7)$, as one subject had sustained a re-rupture (four months after reconstruction, due to a new trauma) and two subjects were lost to follow-up as they moved away from the Groningen region. The first measurements from the subjects lost to follow up were included when comparing ACL-deficient knees to contralateral ACL-intact knees $(\mathrm{N}=10)$. Patient characteristics and measured PTS values are presented in Table 1.

Intraobserver reliability for the medial PTS showed an ICC of 0.82 for observer 1 and 0.83 for observer 2. For the lateral PTS, the ICC for intraobserver reliability was 0.39 for observer 1 and 0.30 for observer

Table 1 Patient Characteristics and PTS values

\begin{tabular}{ll}
\hline & Mean (SD) \\
\hline Age & $24(4.4)$ years \\
Total body height & $184(10) \mathrm{cm}$ \\
Total body weight & $81.3(8.9) \mathrm{kg}$ \\
Body mass index & $24.0(2.1) \mathrm{kg} / \mathrm{m}^{2}$ \\
Injury-to-surgery interval & 4.6 months \\
Medial PTS & $-6.7(2.5)$ degrees \\
Lateral PTS & $-5.7(2.0)$ degrees \\
$\triangle$ PTS & $-1.0(3.5)$ degrees \\
\hline
\end{tabular}

$\triangle \mathrm{PTS}=$ difference between medial PTS and lateral PTS. PTS Posterior tibial slope, $S D$ Standard deviation
2. Interobserver reliability for the medial PTS demonstrated an ICC of 0.82 and 0.46 for the lateral PTS.

The mean values for rTR and ATT during the different movements are displayed in Table 2 for the contralateral ACL-intact, the ACL-deficient and the ACL-reconstructed knees. Compared to the contralateral ACL-intact knees, both the ACL-deficient and the ACLreconstructed knees showed no significant difference in terms of ATT and rTR. (see Table 2). As an example, Fig. 1 shows a graph containing the results of the rTR during SLHD both before and after reconstruction.

The Spearman's correlation coefficients are displayed in Tables 3 and 4 respectively. Little (if any) to weak correlations were found between medial PTS, lateral PTS and $\triangle$ PTS and ATT for ACL-deficient or for ACLreconstructed knees in all three dynamic tests. Little (if any) to weak correlations were found for ACL-deficient knees between medial PTS, lateral PTS, and $\triangle \mathrm{PTS}$ and rTR in all three dynamic tests. In ACL-reconstructed knees, these correlations were all moderate-to-strong, except for the correlations between lateral PTS and rTR during level walking and side jump (little (if any) correlation) and medial PTS and rTR during level walking (weak correlation).

It must be noted that the results of the Spearman's correlation test showed non-significant results, as shown in Tables 3 and 4 .

\section{Discussion}

Our study aimed to examine whether PTS is correlated to either ATT or rTR during high-demand activities. The main finding was little (if any) to weak correlation between dynamic ATT and PTS, both before and after ACL reconstruction. By studying subjects using an in vivo motion-capture system, the dynamic forces of the muscles surrounding the knee joint were enabled, in contrast to what happens when measuring passive ATT.

Table 2 rTR and ATT during different movements in ACL-deficient, ACL-reconstructed and ACL-intact knees

\begin{tabular}{|c|c|c|c|}
\hline Kinematic variable & ACL-deficient & ACL-reconstructed & ACL-intact \\
\hline \multicolumn{4}{|c|}{ Range of tibial rotation (in degrees; mean (SD)) } \\
\hline Level walking & $13.7(4.1)^{a}(P=0.15, n s)$ & $14.1(3.9)^{b}(P=0.12, n s)$ & $17.3(6.4)$ \\
\hline SLHD & $16.9(3.7)^{a}(P=0.21, \mathrm{~ns})$ & $18.4(3.4)^{b}(P=0.64, n s)$ & $19.4(5.5)$ \\
\hline Side jump & $16.6(5.8)^{a}(P=0.08, n s)$ & $18.3(4.7)^{b}(P=0.24, n s)$ & $20.7(3.6)$ \\
\hline \multicolumn{4}{|c|}{ Anterior tibial translation (in mm; mean (SD)) } \\
\hline Level walking & $4.6(4.8)^{a}(P=0.13, n s)$ & $4.8(5.4)^{b}(P=0.25, n s)$ & $6.6(3.0)$ \\
\hline SLHD & $9.3(5.1)^{a}(P=0.21, \mathrm{~ns})$ & $11.7(9.2)^{b}(P=0.60, n s)$ & $13.4(7.2)$ \\
\hline Side jump & $6.7(5.5)^{a}(P=0.65, n s)$ & $8.8(7.5)^{b}(P=0.78, n s)$ & $7.7(5.8)$ \\
\hline
\end{tabular}

a paired t-test results comparing the $\mathrm{ACL}$-deficient knee to the contralateral $\mathrm{ACL}$-intact knee

${ }^{b}$ paired t-test results comparing the $A C L$-reconstructed knee to the contralateral $A C L$-intact knee

ACL Anterior cruciate ligament, SLHD Single-leg hop for distance, SD Standard deviation, mm Millimeter, ns Non-significant result 


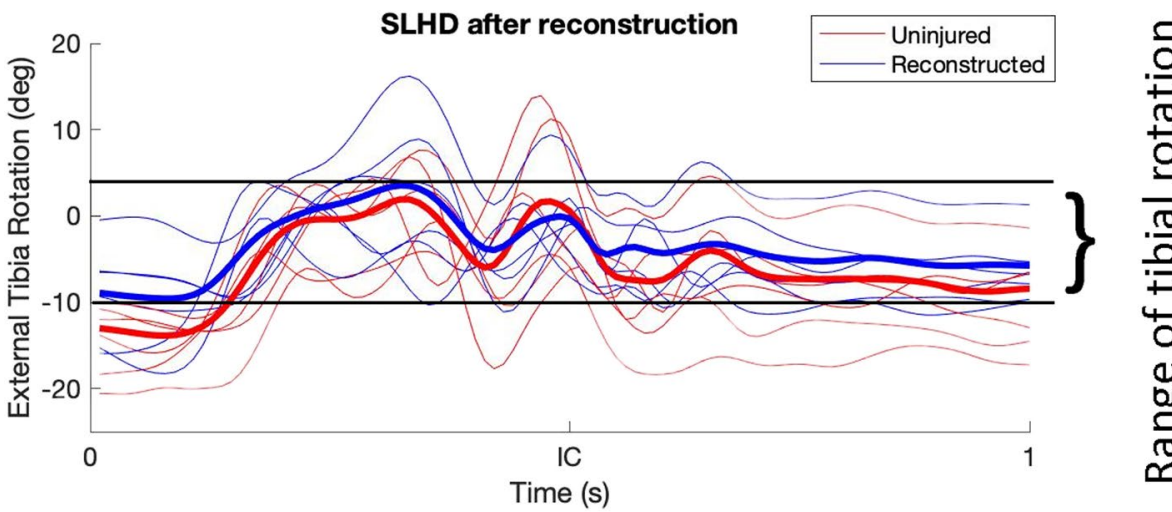

Fig. 1 Example of results regarding the range of tibial rotation in both ACL injured knees (red lines) and ACL reconstructed knees (blue lines). The averages are depicted using the bold red and blue line respectively, The solid black lines represent the upper and lower limit of the range of rotation, in this example from the ACL injured knees

Table 3 Spearman's correlation coefficient and significance level for the correlation between ATT and different types of slope

\begin{tabular}{|c|c|c|}
\hline & \multicolumn{2}{|c|}{$\begin{array}{l}\text { Spearman Correlation Coefficient }(\rho) \\
\text { (Significance level (P)) }\end{array}$} \\
\hline & ACL-deficient & ACL-reconstructed \\
\hline \multicolumn{3}{|c|}{ ATT and medial PTS } \\
\hline Level walking & $\rho=-0.19(P=0.60, n s)$ & $\rho=-0.07(P=0.88, n s)$ \\
\hline SLHD & $\rho=-0.13(P=0.73, n s)$ & $\rho=-0.14(P=0.76, n s)$ \\
\hline Side jump & $\rho=-0.18(P=0.63$, ns $)$ & $\rho=-0.18(P=0.70, n s)$ \\
\hline \multicolumn{3}{|c|}{ ATT and lateral PTS } \\
\hline Level walking & $\rho=0.08(\mathrm{P}=0.83, \mathrm{~ns})$ & $\rho=0.29(\mathrm{P}=0.54, \mathrm{~ns})$ \\
\hline SLHD & $\rho=0.44(\mathrm{P}=0.20, \mathrm{~ns})$ & $\rho=-0.11(P=0.82, \mathrm{~ns})$ \\
\hline Side jump & $\rho=0.25(\mathrm{P}=0.49, \mathrm{~ns})$ & $\rho=0.18(\mathrm{P}=0.70, \mathrm{~ns})$ \\
\hline \multicolumn{3}{|l|}{ ATT and $\triangle P T S$} \\
\hline Level walking & $\rho=-0.26(P=0.47, \mathrm{~ns})$ & $\rho=-0.39(P=0.38, n s)$ \\
\hline SLHD & $\rho=-0.47(\mathrm{P}=0.17, \mathrm{~ns})$ & $\rho=-0.04(P=0.94, n s)$ \\
\hline Side Jump & $\rho=-0.46(P=0.19, \mathrm{~ns})$ & $\rho=-0.43(P=0.34$, ns $)$ \\
\hline
\end{tabular}

ACL Anterior cruciate ligament, SLHD Single-leg hop for distance, SD Standard deviation, PTS Posterior tibial slope, ns Non-significant result

The influence of muscle activity may have led to a weak correlation between PTS and dynamic ATT in our study. Earlier studies show a correlation between PTS and ATT in a passive situation, and particularly that an increase in PTS leads to increased passive ATT [8-10, 23, 24, 27, $28,33,34]$. This previously observed correlation between PTS and passive ATT might be the sole representation of the mechanical interaction between the femur and the tibial slope, as explained by Dejour and Bonnin [10]. Our study suggests that muscular activity enables subjects to compensate for anatomical factors such as PTS by moderating their muscle activation patterns and kinematics when studied during high-demand activities. Dynamic ATT, as measured in our study, is clinically more relevant than passive ATT, as the clinical feeling of giving way is experienced during high-demand activities.

Muscle forces may be able to reduce dynamic ATT in ACL deficiency and after ACL reconstruction. We indeed found that the measured values for both dynamic rTR and ATT seemed lower in ACL-deficient knees and ACL-reconstructed knees compared to their contralateral intact limbs, although this difference was not significant. This may be explained by reduced quadriceps activity of the injured limb, which increases hamstringsto-quadriceps ratio (HQ ratio). As shown in a 3D computer model by Shelburne et al., reducing quadriceps force can lower ATT in the presence of ACL deficiency [29]. This theory is referred to as the quadriceps avoidance pattern. Moreover, computer models showed that an increase in hamstrings activity, also leading to an increased HQ ratio, likewise reduces the dynamic ATT $[29,31]$. Although the theory of altered muscle activation to reduce dynamic ATT is supported by several authors [2, 16, 21, 29], it has been refuted by Keizer et al. [18], who studied healthy subjects with an intact ACL in vivo. In their study, subjects with lax knees on instrumented Lachman displayed less dynamic ATT during SLHD than subjects with lower ATT on instrumented Lachman. Electromyography (EMG) obtained during the SLHD landing showed no clear relation between muscle activity patterns and dynamic ATT, yet less knee flexion was shown by subjects with lax knees. Keizer et al. concluded that landing kinematics may be more relevant than muscle activation in controlling dynamic ATT. Chmielewski et al. found landing kinematics comparable to Keizer et al., i.e. less knee flexion, in subjects with acute ACL injury [5]. In our study these landing kinematics were not 
Table 4 Spearman's correlation coefficient and significance level for the correlation between rTR and different types of slope

\begin{tabular}{|c|c|c|}
\hline & \multicolumn{2}{|c|}{ Spearman Correlation Coefficient $(\rho)$ (Significance level (P)) } \\
\hline & ACL-deficient & ACL-reconstructed \\
\hline \multicolumn{3}{|c|}{ Range of tibial rotation and medial PTS } \\
\hline Level walking & $\rho=-0.21(P=0.56, \mathrm{~ns})$ & $\rho=-0.39(P=0.38, \mathrm{~ns})$ \\
\hline SLHD & $\rho=0.48(P=0.16, \mathrm{~ns})$ & $\rho=0.64(P=0.12, \mathrm{~ns})$ \\
\hline Side Jump & $\rho=0.44(P=0.20, \mathrm{~ns})$ & $\rho=0.69(P=0.06, n s)$ \\
\hline \multicolumn{3}{|c|}{ Range of tibial rotation and lateral PTS } \\
\hline Level walking & $\rho=-0.50(P=0.14, n s)$ & $\rho=-0.04(P=0.94, n s)$ \\
\hline SLHD & $\rho=0.10(\mathrm{P}=0.78, \mathrm{~ns})$ & $\rho=0.54(\mathrm{P}=0.22, \mathrm{~ns})$ \\
\hline Side Jump & $\rho=0.08(P=0.83, n s)$ & $\rho=-0.14(P=0.74, \mathrm{~ns})$ \\
\hline \multicolumn{3}{|c|}{ Range of tibial rotation and $\triangle P T S$} \\
\hline Level walking & $\rho=0.21(P=0.56, \mathrm{~ns})$ & $\rho=-0.50(P=0.25, \mathrm{~ns})$ \\
\hline SLHD & $\rho=0.32(P=0.41, \mathrm{~ns})$ & $\rho=-0.64(P=0.12, \mathrm{~ns})$ \\
\hline Side Jump & $\rho=0.37(\mathrm{P}=0.29, \mathrm{~ns})$ & $\rho=0.71(P=0.05, \mathrm{~ns})$ \\
\hline
\end{tabular}

ACL Anterior cruciate ligament, SLHD Single-leg hop for distance, SD Standard deviation, PTS Posterior tibial slope, $n$ s Non-significant result

seen; no significant difference was observed in maximum knee flexion or knee extension between ACL-intact and ACL-deficient knees.

Several compensation techniques may be successful in reducing dynamic ATT, such as altering landing kinematics or altering muscle activation patterns. A subject's (biomechanical or anatomical) profile may result in preference for a compensation technique, but most likely it is a complex interplay of many factors. A 3D model fed with material properties, geometrical data, and experimental data (kinematics and EMG data) during dynamic tasks may provide more insight into possible compensation techniques to reduce dynamic ATT. Factors such as self-efficacy, psychological readiness, and subjective knee function may also play an important role. As shown in our earlier work (Zee et al.), psychological readiness and subjective knee function are related to dynamic rTR in ACL deficiency and after ACL reconstruction.

This study is the first to explore a correlation between PTS and dynamic rTR. As with dynamic ATT, little (if any) to weak correlations between dynamic rTR and PTS were observed in ACL deficiency. More specifically, little (if any) to weak correlations were found between dynamic $\mathrm{rTR}$ and $\triangle \mathrm{PTS}$ in ACL deficiency. In acute ACL injury, similarly to the mechanism involved in reducing ATT, diminished hamstring muscle activity has shown to be related to decreased internal rotation of the tibia in ACL-reconstructed subjects [1]. This emphasizes the possibility of the hamstrings influencing rTR, and in doing so, counteracting the influence of PTS on rTR in acute ACL deficiency. However, one year after ACL reconstruction we have observed moderate-to-strong correlations between rTR and PTS. This may indicate that the previously hypothesized compensation mechanisms fail to compensate for rotatory laxity in the long run. Taking these factors into account, caution should be exercised with highly invasive procedures such as an anterior closing-wedge osteotomy of the tibia. Theoretically, a tibial osteotomy will influence the biomechanical interaction between passive ATT and PTS but neglects the (powerful) influence of muscle activation. Ultimately, the correlation between PTS and ATT may be corrected by muscle activation, but this may not be the case for the correlation between PTS and rTR. Hence the possibilities of an alternative osteotomy technique to correct for tibial rotation, for instance an anteromedial opening wedge, may be explored.

\section{Limitations and future research}

This study has several limitations. The narrow inclusion and exclusion criteria were mainly responsible for the small sample size-for instance, subjects with concomitant injury were excluded. Injury to the menisci and anterolateral structures of the knee are known to influence the amount of tibial rotation [20]. By including subjects with concomitant injury, the results could have been biased. Although concomitant injury is a common feature in the general population, we regard our results as an accurate representation of the biomechanics involved in solitary ACL deficiency. The limited sample size is mainly responsible for the non-significant result of the correlation tests. However correlation coefficients are more relevant when interpreting Spearman's test as opposed to significance levels. Nonetheless the results our study urge the need for future studies with more subjects to confirm the correlations found. Our study did not include 
electromyography (EMG) measurements to support our theory. In future research it would be interesting to incorporate the use of EMG to evaluate muscle activation patterns during SLHD in ACL deficiency and after ACLR.

The average medial PTS in our population was $-6.7^{\circ}$ (95\% CI $-4.9 ;-8.5)$, and in the lateral compartment $-5.7^{\circ}$ (95\% CI $-4.3 ;-7.1)$. It must be noted that interobserver and intraobserver agreement was poorer for lateral PTS compared to medial PTS. Still, our observed PTS values are comparable to previous studies. In a systematic review and meta-analysis by Wordeman et al., average lateral PTS in ACL-injured subjects was between -1.8 $( \pm 3.2)$ and $-11.5( \pm 3.54)$ degrees [35]. Average medial PTS in ACL-injured subjects was between $+1.8( \pm 3.5)$ and $-12.1( \pm 3.3)$ degrees.[35].

We cannot state whether the aforementioned compensation mechanisms are able to limit ATT in subjects with higher levels of PTS. Dejour et al. report a significant increase of passive ATT with PTS $>12^{\circ}[8]$, Li et al. report increased passive ATT with PTS of $10^{\circ}$ and Webb et al. report increased risk of ACL injury and graft failure with PTS $>12^{\circ}[23,34]$. Observed PTS did not reach these values in our population. It would be of interest to additionally investigate the relation between PTS and ATT during in vivo motion. The $\triangle \mathrm{PTS}$ variable is theoretically interesting to explore further with respect to tibial rotation.

\section{Conclusions}

In contrast to passive ATT, which is significantly correlated to PTS, little (if any) to weak correlations were found between dynamic ATT and PTS. A compensation mechanism seems to be able to correct for the anatomical influence of PTS on dynamic ATT during high-demand tasks. Moderate-to-strong correlations between PTS and dynamic rTR were found one year after ACL reconstruction. These findings warrant prudence in the use of a pure anterior closing-wedge osteotomy in ACL reconstruction; the effect of an anteromedial opening wedge on dynamic ATT and rTR may be further explored.

\section{Abbreviations \\ ACL: Anterior cruciate ligament; ATT: Anterior tibial translation; EMG: Elec- tromyography; PTS: Posterior tibial slope; rTR: Range of tibial rotation; SLHD: Single-leg hop for distance; SD: Standard deviation.}

\section{Acknowledgements}

The authors would like to thank Dr. CMI Beijersbergen and R. Hensen for their aid in data collection.

\section{Authors' contributions}

$M Z$, JVR, JH and RD were involved in designing the study. MZ, MK, JVR and RD contributed to data collection and processing. The data was analyzed by MZ and LD. Both MZ and LD were involved in drafting the manuscript, which was critically reviewed by MK, JVR, JH, and RD. The authors read and approved the final manuscript.
Funding

No funding was received for this study.

Availability of data and materials

All data is available from the corresponding author upon reasonable request.

\section{Declarations}

Ethics approval and consent to participate

IRB UMCG (ID 2015/524), informed consent was provided by all included subjects.

\section{Consent for publication}

Not applicable.

\section{Competing interests}

The authors declare no competing interest.

\section{Author details}

${ }^{1}$ Department of Orthopaedic Surgery, University Medical Center Groningen, PO Box 30.001, 9700 RM Groningen, The Netherlands. ${ }^{2}$ Department of Human Movement Science, University Medical Center Groningen, University of Groningen, Groningen, The Netherlands. ${ }^{3}$ Department of Orthopaedic Surgery, Martini Hospital, Groningen, The Netherlands. ${ }^{4}$ Department of Rehabilitation Medicine, University Medical Center Groningen, Groningen, The Netherlands.

Received: 22 June 2021 Accepted: 13 August 2021

Published online: 02 September 2021

\section{References}

1. Abourezk MN, Ithurburn MP, McNally MP, Thoma LM, Briggs MS, Hewett TE, Spindler KP, Kaeding CC, Schmitt LC (2017) Hamstring strength asymmetry at 3 years after anterior cruciate ligament reconstruction alters knee mechanics during gait and jogging. Am J Sports Med 45:97-105

2. Barcellona MG, Morrissey MC, Milligan P, Amis AA (2014) The effect of thigh muscle activity on anterior knee laxity in the uninjured and anterior cruciate ligament-injured knee. Knee Surg Sports Traumatol Arthrosc 22:2821-2829

3. Bernhardson AS, Aman ZS, Dornan GJ, Kemler BR, Storaci HW, Brady AW, Nakama GY, LaPrade RF (2019) Tibial slope and its effect on force in anterior cruciate ligament grafts: anterior cruciate ligament force increases linearly as posterior tibial slope Increases. Am J Sports Med 47:296-302

4. Boeth $H$, Duda GN, Heller MO, Ehrig RM, Doyscher R, Jung T, Moewis $P$, Scheffler S, Taylor WR (2013) Anterior cruciate ligament-deficient patients with passive knee joint laxity have a decreased range of anterior-posterior motion during active movements. Am J Sports Med 41:1051-1057

5. Chmielewski TL, Rudolph KS, Fitzgerald GK, Axe MJ, Snyder-Mackler L (2001) Biomechanical evidence supporting a differential response to acute ACL injury. Clin Biomech (Bristol, Avon) 16:586-591

6. Christensen JJ, Krych AJ, Engasser WM, Vanhees MK, Collins MS, Dahm DL (2015) Lateral tibial posterior slope is increased in patients with early graft failure after anterior cruciate ligament reconstruction. Am J Sports Med 43:2510-2514

7. Davis RB III, Ounpuu S, Tyburski D, Gage JR (1991) A gait analysis data collection and reduction technique. Hum Mov Sci 10:575-587

8. Dejour D, Pungitore M, Valluy J, Nover L, Saffarini M, Demey G (2019) Preoperative laxity in ACL-deficient knees increases with posterior tibial slope and medial meniscal tears. Knee Surg Sports Traumatol Arthrosc 27:564-572

9. Dejour D, Pungitore M, Valluy J, Nover L, Saffarini M, Demey G (2019) Tibial slope and medial meniscectomy significantly influence short-term knee laxity following ACL reconstruction. Knee Surg Sports Traumatol Arthrosc 27:3481-3489

10. Dejour H, Bonnin M (1994) Tibial translation after anterior cruciate ligament rupture. Two radiological tests compared. J Bone Joint Surg Br 76:745-749

11. Domholdt E (2000) Physical therapy research: Principles and applications, 2nd edn. W B Saunders Co, Philadelphia 
12. Fu FH, Bennett CH, Ma CB, Menetrey J, Lattermann C (2000) Current trends in anterior cruciate ligament reconstruction. Part II. Operative procedures and clinical correlations. Am J Sports Med 28:124-130

13. Giffin JR, Vogrin TM, Zantop T, Woo SL, Harner CD (2004) Effects of increasing tibial slope on the biomechanics of the knee. Am J Sports Med 32:376-382

14. Gornitzky AL, Lott A, Yellin JL, Fabricant PD, Lawrence JT, Ganley TJ (2016) Sport-specific yearly risk and incidence of anterior cruciate ligament tears in high school athletes: a systematic review and meta-analysis. Am J Sports Med 44:2716-2723

15. Hudek R, Schmutz S, Regenfelder F, Fuchs B, Koch PP (2009) Novel measurement technique of the tibial slope on conventional MRI. Clin Orthop Relat Res 467:2066-2072

16. Hurd WJ, Snyder-Mackler L (2007) Knee instability after acute ACL rupture affects movement patterns during the mid-stance phase of gait. J Orthop Res 25:1369-1377

17. Imhoff FB, Mehl J, Comer BJ, Obopilwe E, Cote MP, Feucht MJ, Wylie JD, Imhoff AB, Arciero RA, Beitzel K (2019) Slope-reducing tibial osteotomy decreases ACL-graft forces and anterior tibial translation under axial load. Knee Surg Sports Traumatol Arthrosc 27:3381-3389

18. Keizer MNJ, Hijmans JM, Gokeler A, Benjaminse A, Otten E (2020) Healthy subjects with lax knees use less knee flexion rather than muscle control to limit anterior tibia translation during landing. J Exp Orthop 7:32-020-00246-6

19. Keizer MNJ, Otten E (2020) Technical note: sensitivity analysis of the SCORE and SARA methods for determining rotational axes during tibiofemoral movements using optical motion capture. J Exp Orthop 7:6-020-0219-z

20. Kittl C, El-Daou H, Athwal KK, Gupte CM, Weiler A, Williams A, Amis AA (2016) The role of the anterolateral structures and the ACL in controlling laxity of the intact and ACL-deficient knee: response. Am J Sports Med 44:NP15-8

21. Klyne DM, Keays SL, Bullock-Saxton JE, Newcombe PA (2012) The effect of anterior cruciate ligament rupture on the timing and amplitude of gastrocnemius muscle activation: a study of alterations in EMG measures and their relationship to knee joint stability. J Electromyogr Kinesiol 22:446-455

22. Koo TK, Li MY (2016) A guideline of selecting and reporting intraclass correlation coefficients for reliability research. J Chiropr Med 15:155-163

23. Li Y, Hong L, Feng H, Wang Q, Zhang J, Song G, Chen X, Zhuo H (2014) Posterior tibial slope influences static anterior tibial translation in anterior cruciate ligament reconstruction: a minimum 2-year follow-up study. Am J Sports Med 42:927-933
24. Marouane H, Shirazi-Adl A, Hashemi J (2015) Quantification of the role of tibial posterior slope in knee joint mechanics and ACL force in simulated gait. J Biomech 48:1899-1905

25. Musahl V, Karlsson J (2019) Anterior cruciate ligament tear. N Engl J Med 380:2341-2348

26. Robertson D, Caldwell G, Hamill J, Kamen G, Whittlesey S (2013) Research methods in biomechanics, 2nd edn. ((eBook))

27. Sauer S, Clatworthy M (2018) The effect of medial tibial slope on anterior tibial translation and short-term ACL reconstruction outcome. Surg J (N Y) 4:e160-e163

28. Schneider A, Arias C, Bankhead C, Gaillard R, Lustig S, Servien E (2020) Greater medial tibial slope is associated with increased anterior tibial translation in females with an ACL-deficient knee. Knee Surg Sports Traumatol Arthrosc 28:1901-1908

29. Shelburne KB, Torry MR, Pandy MG (2005) Effect of muscle compensation on knee instability during ACL-deficient gait. Med Sci Sports Exerc 37:642-648

30. Shen L, Jin ZG, Dong QR, Li LB (2018) Anatomical risk factors of anterior cruciate ligament injury. Chin Med J (Engl) 131:2960-2967

31. Shimokochi Y, Shultz SJ (2008) Mechanisms of noncontact anterior cruciate ligament injury. J Athl Train 43:396-408

32. Todd MS, Lalliss S, Garcia E, DeBerardino TM, Cameron KL (2010) The relationship between posterior tibial slope and anterior cruciate ligament injuries. Am J Sports Med 38:63-67

33. Tradati D, Mouton C, Urhausen A, Beel W, Nuhrenborger C, Seil R (2020) Lateral meniscal slope negatively affects post-operative anterior tibial translation at 1 year after primary anterior cruciate ligament reconstruction. Knee Surg Sports Traumatol Arthrosc 2020;28(11):3524-3531. https://doi.org/10.1007/s00167-020-06021-5

34. Webb JM, Salmon LJ, Leclerc E, Pinczewski LA, Roe JP (2013) Posterior tibial slope and further anterior cruciate ligament injuries in the anterior cruciate ligament-reconstructed patient. Am J Sports Med 41:2800-2804

35. Wordeman SC, Quatman CE, Kaeding CC, Hewett TE (2012) In vivo evidence for tibial plateau slope as a risk factor for anterior cruciate ligament injury: a systematic review and meta-analysis. Am J Sports Med 40:1673-1681

\section{Publisher's Note}

Springer Nature remains neutral with regard to jurisdictional claims in published maps and institutional affiliations.

\section{Submit your manuscript to a SpringerOpen ${ }^{\circ}$ journal and benefit from:}

- Convenient online submission

- Rigorous peer review

- Open access: articles freely available online

- High visibility within the field

- Retaining the copyright to your article

Submit your next manuscript at springeropen.com 